\title{
Information Gathering and Marketing ${ }^{1}$
}

\author{
Heski Bar-Isaac
}

NYU

\author{
Guillermo Caruana
}

CEMFI

April, 2008

\author{
Vicente Cuñat
}

LSE

\begin{abstract}
Consumers have only partial knowledge before making a purchase decision, but can choose to acquire more detailed information. A firm can make it easier or harder for these consumers to obtain such information. We explore consumers' information gathering and the firm's integrated strategy for marketing, pricing, and investment in quality. In particular, we highlight that when consumers are ex-ante heterogeneous, the firm might choose an intermediate marketing strategy for two quite different reasons. First, it serves as a non-price means of discrimination - it can make information only partially available, in a way that induces some, but not all, consumers to acquire the information. Second, when the firm cannot commit to a given investment in quality, it can still convince all consumers of its provision by designing a pricing and marketing policy that induces some consumers to actively gather further information. This mass of consumers, in exchange, is sufficiently large to discipline the monopolist to invest in the quality of the product.
\end{abstract}

JEL: D42, D83, L15, M31

Keywords: information gathering, monopoly, marketing, pricing, investment

\footnotetext{
${ }^{1}$ We thank participants at EARIE 2007 (Valencia), IIOC 2007 (Savannah), LSE, Michigan State University, Stern Marketing lunch, Stern Micro lunch, University of Sydney, Utah Winter Business Economics Conference, Simon Anderson, Simon Board, Jim Dana, Andrew Daughety, Hao Li, Regis Renault, and, particularly, Yuk-Fai Fong and Monic Jiayin Sun for detailed and helpful comments.

Contact info: Bar-Isaac: heski@nyu.edu; Department of Economics, Stern School of Business, NYU, 44 West 4th street 7-73, NYC, NY 10012 USA; Caruana: caruana@cemfi.es; Casado del Alisal 5, 28014 Madrid, Spain; and Cuñat: v.cunat@lse.ac.uk; Department of Finance, London School of Economics, Houghton Street, London WC2 2AE, UK.
} 


\section{Introduction}

Before deciding whether to buy a good or service, consumers often have the opportunity to gather information or simply spend time thinking about how much they would enjoy the good. Gathering or processing information is costly, in terms of money, time and effort. A firm, through its advertising, product design, and marketing strategies, can affect these costs and make it easier or harder for consumers to assess whether a product is a good match for their needs or preferences. In this paper, we explore a monopolist firm's marketing strategy by characterizing the firm's choice of how costly it is for consumers to learn their valuations of the good. The marketing decision, of course, interacts with the firm's investment in quality and its pricing decision.

To take a specific example, a firm selling software determines prices and how much to invest in development. It can also choose how easy it is for customers to figure out their valuation for the software before they purchase it: The firm could simply list or advertise some of the applications and features; it could, additionally, illustrate these through describing the performance in standard tasks; or it could even allow trial versions that permit potential consumers to try the product for a period. Consumers initially have some idea of how much the software might be worth to them, but the access to additional information would allow them to research further, revise their opinions, and attain a more precise valuation of the software.

If consumers could fully inspect the good, they would still perceive it differently, due to idiosyncratic taste differences. From the firm's perspective, making it easier for consumers to learn their valuations will have the positive effect that some of them will be willing to pay a relatively high price when they learn that the product is a good match for them; but, also, the negative effect that some others learn that the product is a bad match and their willingness to pay is accordingly reduced.

When consumers are ex-ante identical in their expectations about the good, this trade-off resolves itself to one extreme or the other. Either the firm prefers to make it impossible for consumers to learn their valuations, choosing an opaque policy, and sells with probability one at the average valuation, or else it chooses a transparent policy and sells to those with high realized valuation at high prices. This is precisely the trade-off between a broad, full-market strategy or a niche-targeting one. Similar considerations have been described, for example, in Lewis and Sappington (1994) and 
Johnson and Myatt (2006). ${ }^{2}$ Further, it can readily be shown that if marginal costs of production are higher, the costless information (niche) strategy is more likely to be preferred.

However, if consumers are ex-ante heterogeneous (if a good match is worth more to some consumers than others), the firm might prefer an intermediate information strategy. In this case, some consumers choose to get informed, while others prefer to buy without getting informed. Indeed, the firm might prefer an intermediate information strategy even if, when dealing with each type separately, it would use the same extreme policy. In particular, a firm might pursue the same marketing strategy in two different markets, but, following integration of these markets, choose a different strategy for the combined market.

This result can arise for two different reasons. First, the firm's marketing strategy is integrated with its pricing strategy; therefore, when dealing with ex-ante heterogeneous consumers, an intermediate marketing strategy can act as a non-price means of discriminating between different consumer types. Highly interested consumers prefer to buy immediately, without any extra information, while less interested consumers buy only after having checked for quality. Second, an intermediate marketing strategy can also serve as an indirect form of commitment to provide quality. When some consumers verify the quality of the good and buy conditional on what they observe, they implicitly act as monitors for the rest of the consumers, who can buy without assessing. In other words, those assessing give the firm sufficiently strong incentives to invest in quality, even when this investment is not directly observable. This is important, for example, in the case of a new firm without an established reputation for the quality of its product.

We are able to illustrate these two considerations in a fairly general model. We then move on to examine a particular specification of the model in which tastes are linear functions of consumer types, types are distributed uniformly, and investment in quality is a discrete decision. Within this structure, the mechanisms at work can be observed easily. Furthermore, we prove that an established firm that can commit to a level of investment in quality is more likely to choose an intermediate marketing strategy when high-value consumers are relatively insensitive to the idiosyncratic match quality.

Our approach and discussion complement some recent work on the economics of advertising that is in contrast to much of the earlier literature (see Bagwell, 2007, for an excellent and thorough

\footnotetext{
${ }^{2}$ See, also, Creane (2008) for a recent and interesting application of this intuition.
} 
survey). In particular, we explain the diversity of advertising and marketing strategies by focusing on the informational content of advertising and its strategic use. We abstract from the more traditional views of advertising as a costly signaling device, or that advertising enters into preferences directly. Closest in terms of the question and model of this paper is Zettelmeyer (2000); however, there, the primary concern is competition, and so the model makes some restrictions in other respects. In particular, it assumes that customers are identical ex-ante; as a consequence, with a monopoly provider, agents never pay to gather information in equilibrium, in contrast to a central result and intuition in our paper. Further, Zettelmeyer does not consider the firm's commitment to investment - another central concern of our work. Anderson and Renault (2006) show that an intermediate information policy can often be optimal in a model in which consumers are passive, in the sense that they take no active information gathering role. In their setup, the optimality of intermediate information relies on overcoming the holdup associated with the costs of going to the store (the Diamond paradox) and so arises through a very different channel from the one we discuss. Johnson and Myatt (2006) also consider information provision to consumers, but work with an aggregate demand function, and, so, do not consider individual consumers' decisions and cannot identify many of the mechanisms that we discuss. Anand and Shachar (2005) consider the role of advertising in affecting a consumer's beliefs about match quality both theoretically and empirically. Sun (2007) examines how the extent of (known) vertical quality affects a firm's decision to release information about horizontal attributes. Finally, in related work, Bar-Isaac, Caruana, and Cuñat (2008) explore a multidimensional good setting in which consumers also gather information, but do so attribute by attribute. The study suggests that firms have strong incentives to influence the consumers' assessment behavior.

Outside of the literature on branding and advertising, our work is related to Courty and Li (1999, 2000 ), in which the information that consumers have about their valuation for a good increases (exogenously) over time. A firm can exploit this by charging different prices at different times or can offer a menu of refund contracts. Their work nicely characterizes the impact and the comparative statics of different information structures for the consumer types. Our work differs from this and other work on information disclosure, in a number of respects. First, and most significantly, we allow no discrimination through prices: There is only one "contract" offered and all products are sold at an identical price. Second, our consumers are active in information gathering: They choose 
whether or not to incur a cost in learning their valuations and the firm chooses this cost directly. ${ }^{3,4}$

\section{Base Model}

We consider a firm that decides: (i) how much to investment in quality for a single good; (ii) the price of the good; and (iii) the ease with which consumers can learn their valuations for it. Consumers have expectation of how much they are likely to value the good based on how much the firm has invested or, in the case where the firm cannot commit to a given quality provision, on their inferences of how much the firm has invested. Consumers' valuation of the good depends on their type and an idiosyncratic component. We model investment in quality as leading to a product that is more likely to appeal to a broader range of consumers of any type. By incurring some effort that depends on the firm's marketing strategy, a consumer can learn her realized valuation before deciding whether or not to buy.

For the time being, we suppose that investment is observed by consumers, and later, in Section 5 ,we consider the case in which it is not. The specific timing is, therefore, as follows. First, the firm decides on marketing, price, and investment strategy. Consumers observe all these choices and decide whether to acquire more information on the product and, subsequently, whether to buy it.

\section{$2.1 \quad$ Firm}

A monopoly produces a single product incurring a production cost $c(q)$ to obtain $q$ units. The product can be a good or a bad match for each consumer, and this is determined stochastically. The firm can invest a variable amount $x$ to affect the probability that its product becomes a good match for consumers. In particular, any consumer has a probability of finding a good match of $\gamma(x) \in[0,1]$, where $\gamma$ is non-decreasing function.

In addition to choosing its investment strategy, the firm posts a price $p$ for the good, and, costlessly, chooses a marketing strategy $A \in \mathbb{R}^{+} .{ }^{5}$ Consumers can choose to incur a cost $A$ to learn the realization of their valuations before buying the good. We will refer to transparency, when the

\footnotetext{
${ }^{3}$ There is a wide literature that has considered information gathering and more general price mechanisms. See Cremer and Khalil (1992), Lewis and Sappington (1997), Cremer et al. (1998a,b), and Bergemann and Välimäki (2002) or, in the context of auctions, Ganuza and Penalva (2006) and references therein.

${ }^{4}$ Matthews and Persico (2005) study refund policies but their work is related to this paper inasmuch as they do so in a framework with information acquisition, and posted prices. Posted prices and fully flexible disclosures of partial information with passive consumers have been also considered in Saak (2006).

${ }^{5}$ We could also allow $A<0$; however, in this model, consumers will choose to get informed if $A=0$ and there is no advantage to further encourage them.
} 
firm makes it costless for consumers to learn their valuation $(A=0)$. When the firm makes it prohibitively costly ( $A=\infty$ or equivalently an $A$ that is high enough so that no consumer verifies), we will refer to it as opacity. Finally, an intermediate marketing strategy corresponds to those interior choices of $A$ in which some consumers pay to learn the realization of their valuation. Introducing costs to the firm for choosing different marketing strategies would be a natural extension; however, we abstract from it to highlight the economic forces at work. ${ }^{6}$

Summarizing, the firm in this model is risk-neutral and chooses $A, p$, and $x$ to maximize its profits.

\subsection{Consumers}

There is a mass one of consumers, each of whom is potentially interested in buying one unit of the good. Consumers have a taste for quality represented by $\theta \in[0,1]$, where type, $\theta$, is distributed according to some atomless probability density function $f(\cdot)$. Higher values of $\theta$ correspond to consumers who have higher valuations, on average.

However, the valuation of the good depends not only on $\theta$, but also on some ex-ante unknown idiosyncratic aspect that makes it a good or a bad match for the consumer. The probability that a match is good is $\gamma(x){ }^{7}$ The utility of an agent of type $\theta$ who purchases the good at a price $p$ is $g(\theta)-p$ if it is a good match and $b(\theta)-p$ if it is bad. We assume that $g(\theta) \geq b(\theta)$ for all $\theta$ and that $g(\theta)$ and $b(\theta)$ are non-decreasing in $\theta$.

Before purchasing, the agent may decide to assess the quality of the good by spending $A$. There is no point in the agent assessing the quality of the good if she plans to buy the good regardless of the quality level, so assessment will take place only if the subsequent purchase decision is conditional on finding high quality. ${ }^{8}$ In particular, assessment is valuable only as a form of protection or insurance against the possibility of buying a bad match. Therefore, there are only three reasonable strategies for an agent of type $\theta$ and the corresponding expected utilities:

- Buy unconditionally without assessing $E U_{B}(\theta)=\gamma g(\theta)+(1-\gamma) b(\theta)-p$.

- Buy conditionally after assessing $E U_{A}(\theta)=\gamma(g(\theta)-p)-A$.

\footnotetext{
${ }^{6}$ It is not clear how these costs should change. Providing good and accurate information to consumers is costly; but it is also costly to deliberately hide and obfuscate information.

${ }^{7}$ Where there is no ambiguity, we will suppress the argument for $\gamma(x)$ and simply write $\gamma$. Note, also, that the probability of a match is independent of $\theta$.

${ }^{8}$ For expositional purposes, and without loss of generality, we assume that, when $A=0$, consumers that do not condition their purchase on what they see do not assess.
} 
- Not buy (do not assess or buy) $E U_{N}(\theta)=0$.

\section{General Results}

First, we focus on consumer strategies, taking the firm's strategy as given.

\subsection{Characterizing Consumer Behavior}

We begin by introducing two lemmas that allow the behavior of every consumer to be described in a simple way.

Lemma 1 If an agent of type $\theta$ prefers assessing to buying unconditionally, then so do all agents of type $\phi \leq \theta$.

Proof. $\theta$ prefers assessing to buying unconditionally and so

$$
\gamma(g(\theta)-p)-A>\gamma g(\theta)+(1-\gamma) b(\theta)-p
$$

which holds if and only if

$$
p-\frac{A}{1-\gamma}>b(\theta)
$$

Since $b(\theta)$ is non-decreasing in $\theta$, then condition (2) holds for all $\phi \leq \theta$.

Lemma 2 If a consumer of type $\theta$ prefers not to buy, then all consumers with $\phi \leq \theta$ also prefer not to buy.

Proof. $\theta$ prefers not to buy when

$$
0>\max \{\gamma(g(\theta)-p)-A, \gamma g(\theta)+(1-\gamma) b(\theta)-p\} .
$$

Both arguments of the max are non-decreasing in $\theta$, and so condition (3) holds for all $\phi \leq \theta$.

As a consequence of Lemmas 1 and 2, to characterize consumer behavior, it is sufficient to identify the consumers who are indifferent between buying unconditionally and assessing, between buying unconditionally and not buying, and between assessing and not buying. Consumer strategies are homogeneous in the intervals determined by such consumers. ${ }^{9}$ We introduce notation for such

\footnotetext{
${ }^{9}$ Note that, in some circumstances, all consumers may have the same strict preferences over some (or all) of these assessment strategies, so that no consumer is indifferent between two of these strategies.
} 
consumers.

Specifically, let $T_{B A}$ denote the consumer indifferent between buying unconditionally and assessing. Then, $T_{B A}$ is implicitly defined by $E U_{B}\left(T_{B A}\right)=E U_{A}\left(T_{B A}\right)$. By Lemmas 1 and 2 , there can be at most one solution. If there is no solution, it is because all consumers prefer one option over the other. If $E U_{B}(\theta)>E U_{A}(\theta)$ holds for all $\theta$, we define $T_{B A}=0$. This is with some abuse, but has no consequences, as the mass of consumers with $\theta=0$ is zero. When $E U_{B}(\theta)<E U_{A}(\theta)$ holds for all $\theta$, we define in a similar fashion $T_{B A}=1$.

Similarly, we define $T_{B N}$ as the consumer who is indifferent between buying without assessment and not buying. $T_{B A}$ is implicitly defined by the equation $E U_{B}\left(T_{B N}\right)=0$. Again if $E U_{B}(\theta)>0$ for all $\theta$ denote $T_{B N}=0$; and if $E U_{B}(\theta)<0$, then $T_{B N}=1$. Finally, let $T_{A N}$ denote the consumer indifferent between assessing and not buying, implicitly defined by $E U_{A}\left(T_{A N}\right)=0$, and if no solution exists, denote $T_{A N}=0$ if $E U_{A}(\theta)>0$ and $T_{A N}=1$ otherwise.

Note that $T_{B N}, T_{B A}$ and $T_{A N}$ depend on the firm's choice of price, $p$, marketing, $A$, and investment (which appears indirectly through $\gamma$ ), as well as all exogenous parameters of the model; however, we often suppress these arguments for notational simplicity. In the case that $T_{B N}, T_{B A}$ and $T_{A N}$ are interior, then they are implicitly defined as follows:

$$
\begin{aligned}
\gamma g\left(T_{B N}\right)+(1-\gamma) b\left(T_{B N}\right) & =p, \\
b\left(T_{B A}\right) & =p-\frac{A}{1-\gamma}, \\
g\left(T_{A N}\right) & =p+\frac{A}{\gamma} .
\end{aligned}
$$

\subsection{The Firm's Problem}

With these definitions and preliminary results, the firm's sales can be simply written down as:

$$
S=\int_{\max \left\{T_{B N}, T_{B A}\right\}}^{1} f(\theta) d \theta+\gamma 1_{T_{B A}>T_{A N}} \int_{T_{A N}}^{T_{B A}} f(\theta) d \theta
$$

where $1_{T_{B A}>T_{A N}}$ is an indicator function that takes the value 1 if $T_{B A}>T_{A N}$ and 0 otherwise. The first integral in (7) corresponds to sales to consumers who buy without assessment, and the second expression corresponds to those who assess and buy only when they find high quality, which occurs with probability $\gamma$. 
The firm's problem, then, is to choose $(A, p, x)$ in order to maximize profits:

$$
\Pi=p S-c(S)-x,
$$

Note that sales $S$ depend on $T_{B N}, T_{B A}$ and $T_{A N}$ and, therefore, on $(A, p, x)$.

Proposition 3 highlights implications for consumer behavior when the firm optimally chooses an intermediate marketing strategy - that is, $0<A<\infty$ with some consumers assessing, rather than either an opaque $(A=\infty)$ or a transparent $(A=0)$ one.

Proposition 3 Intermediate marketing is strictly optimal only if, in equilibrium, there are both consumers who assess, and consumers who buy without assessment.

Proof. Suppose that the firm's optimal strategy is to choose some intermediate $A \in(0, \infty)$. If all consumers assess, then the firm can do better by increasing the price, and reducing $A$ accordingly (thereby inducing identical assessment and purchase behavior). If no one assesses, then the firm can do no worse by choosing the same price and $A=\infty$.

Proposition 3 illustrates one of the two mechanisms outlined in the introduction. It is at the heart of the idea of using the marketing strategy as a non-price means of discriminating between different consumer types. The proposition suggests (and this is verified in examples below) that the marketing strategy can be profitably used as a means of inducing different consumer types to behave differently.

All of the above has the following implications.

Corollary 4 When intermediate marketing is strictly optimal, there is some interior threshold $T_{B A}$ above which all types buy without assessment and lower types assess and, possibly, another threshold $T_{A N}$ below which consumers do not buy.

Proof. Immediate consequence of Lemmas 1 and 2, and Proposition 3.

Corollary 5 If intermediate marketing is optimal for the firm, there must be variation in the value of a bad match-i.e., b $(\theta)$ cannot be constant. In particular, agents must be heterogeneous.

Proof. By Proposition 3, it is necessary that some agents prefer to assess and others buy without assessment. Suppose that some type $\theta$ prefers to buy without assessment and some type $\phi$ prefers to 
assess. Then, as in (2), it must be that $p-\frac{A}{1-\gamma} \leq b(\theta)$ and $p-\frac{A}{1-\gamma}>b(\phi)$, which would contradict that $b(\theta)$ is constant in $\theta$.

Another necessary condition for intermediate marketing to be optimal is that $b(1)>\min _{q} \frac{c(q)}{q}$. Indeed, if this condition fails, the optimal marketing strategy is either transparency or simply to make no sales. The intuition is clear: intermediate or opaque marketing strategies allow the firm to make sales even when matches are bad. However, if bad matches unambiguously destroy surplus, there is no advantage to making such sales.

Corollaries 4 and 5 contain the main intuition for why intermediate marketing can be used as a means of non-price discrimination. When intermediate marketing is optimal, there is a mass of consumers with high ex-ante valuations of the good (consumers with high $\theta$ ) that buys without assessment. There is also a mass of them with lower ex-ante valuations for the good (lower $\theta$ ) that assesses and buys only upon finding a good match. Finally, there may be a group that has very low ex-ante valuations and decides not to assess or buy. The firm is, therefore, using the marketing strategy as a way to induce consumers with low ex-ante valuations to base their consumption decision on their ex-post valuations. The firm can sell to those with a good idiosyncratic match even if their ex-ante valuation is below the price. At the same time, consumers with high ex-ante valuations remain "in the dark" and base their purchase on their ex-ante valuations. Some of them may eventually find out ex-post that their realized valuation is below the price. ${ }^{10}$

However, the firm cannot directly discriminate between consumers in terms of information, so different assessment behaviors have to be achieved indirectly through the right marketing policy $A$. Assessment can be seen as paying a premium $A$ that insures against a bad realization. Therefore, for some consumers to assess and for some not to, there must be heterogeneity in their valuations upon a bad match. Given that low valuations are increasing in the type, the firm can select an $A$ such that high $\theta$ consumers do not verify, while some low $\theta$ ones do.

It is important to stress that the results so far are fairly general, as they do not depend on the particular choice of consumer utility functions or the type distribution. In the following section, we focus on the family of linear utility functions with uniformly distributed types. This allows us to write explicit expressions for $p$ and $A$, and to gain additional intuition about when each marketing strategy is optimal.

\footnotetext{
${ }^{10}$ In other words, intermediate marketing acts as a broad market strategy with high ex-ante valuation consumers, while it acts as a niche strategy with low ex-ante valuation ones.
} 


\section{The Linear-Uniform Case}

In this section, we make some more-specific assumptions on the model to fully characterize the equilibrium. We demonstrate that intermediate marketing and discrimination can arise, and we explore the role of consumers' preferences for these phenomena to happen. Specifically, suppose that $c(q)=c q$, the distributions of consumers is uniform on $[0,1]$, and valuations are linear in type so that $b(\theta)=b+s \theta$ and $g(\theta)=g+(s+\Delta) \theta$. Suppose, also, that investment is a binary decision and that $\gamma=\frac{1}{2}$ if the firm makes an investment at cost $k$ and $\gamma=0$ otherwise. Note that our earlier assumptions on $b(\theta)$ and $g(\theta)$ require that $g \geq b, s \geq 0, \Delta>(b-g)$ and $s+\Delta \geq 0$.

It is worth noting that $\Delta$ need not be positive; indeed, we contrast the case where $\Delta>0$, corresponding to high-types being relatively more sensitive to quality (since here, $g(\theta)-b(\theta)$ increases in $\theta$ ), and $\Delta<0$, so that higher-types are relatively insensitive to quality. Both assumptions are plausible. For example, one might imagine that higher-income types are both more likely to consume and to have a relatively higher valuation for additional quality. In contrast, if someone has a greater need, he might have higher willingness to pay but be less sensitive to quality (for example, a starving person).

The firm wants to maximize profits by choosing $(A, p, x)$. $>$ From Equations (7) and (8), we can write down the firm's profit function (using the assumption that $\theta$ is uniformly distributed) as:

$$
\Pi=(p-c)\left[\left(1-\max \left\{T_{B N}, T_{B A}\right\}\right)+\gamma\left(T_{B A}-T_{A N}\right) \cdot 1_{T_{B A}>T_{A N}}\right]-k \cdot 1_{\text {invest }}
$$

Given that the investment decision is binary, we treat each case separately. First, we consider the (less interesting) case in which the firm makes no investment. Then, the marketing strategy is irrelevant: consumers never consider assessing, as they have no doubts that the match will be bad. Thus, we can conclude that $T_{B A}=T_{A N}=0$. Using Equation (4), we obtain $T_{B N}=$ $\max \left(\min \left(\frac{p-b}{s}, 1\right), 0\right)$ and profits simplify to $\Pi=(p-c)\left(1-T_{B N}\right)$. Depending on the values of the parameters, the optimal price results in either an interior solution with $p_{N I}^{*}=\frac{b+c+s}{2}$ and profits

of $\Pi_{N I}^{*}=\frac{(B-c+s)^{2}}{4 s}$; or a corner solution of either $p_{N I}^{*}=b$ and $\Pi_{N I}^{*}=b-c$, or $p_{N I}^{*} \geq b+s$ and $\Pi_{N I}^{*}=0$ (which is equivalent to not operating and no sales).

Now, we analyze the more interesting case in which the firm invests in quality. We can characterize consumer behavior in terms of the parameters using Equations (4), (5), and (6), as follows: 


$$
\begin{aligned}
& T_{B N}=\max \left(\min \left(\frac{2 p-g-b}{2 s+\Delta}, 1\right), 0\right), \\
& T_{B A}=\max \left(\min \left(\frac{p-b-2 A}{s}, 1\right), 0\right), \\
& T_{A N}=\max \left(\min \left(\frac{2 A-g+p}{s+\Delta}, 1\right), 0\right) .
\end{aligned}
$$

These are illustrated in Figure 1 for the intermediate case. Note that by assessing rather than always buying, an agent saves the cost of paying a price $p$ that is above his valuation (in case of a low realization). He gains this benefit (equal to $p-b+\theta s$ ) with probability $\frac{1}{2}$, but must pay a cost $A$. Similarly, in assessing rather than never buying, a consumer gains a surplus $\frac{1}{2}(g+(s+\Delta) \theta-p)$ (by buying the well-matched product with probability $\frac{1}{2}$ ), which must outweigh the cost of assessment $A$ (which is always paid) for assessment to be worthwhile.

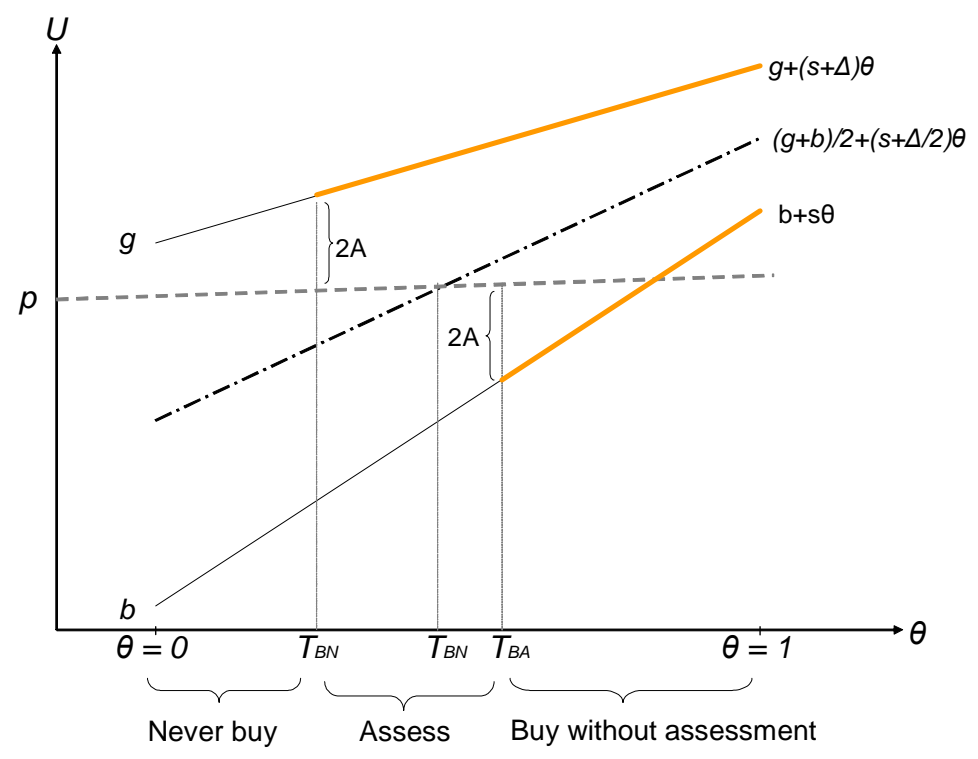

Figure 1: Characterising consumer behavior.

A straight first-order condition approach to obtain the optimal marketing and price choices is cumbersome because of the possibility of corner solutions. Thus, we consider different cases separately, depending on the choice of marketing. In Appendix A, we fully characterize the optimal solutions under transparency $(A=0)$ and opacity $(A=\infty)$. Each of them is a standard monopolist problem with a simple linear demand (piece-wise linear in the case of $A=0$ ). Here, we consider 
the intermediate marketing in detail, as this is the case that best provides intuitions. An optimal intermediate marketing strategy, following Proposition 3, requires $1>T_{B A}>T_{A N} \geq 0$. In this case, we can rewrite the firm's profits from Equation (9) as

$$
\Pi_{\text {Int }}=(p-c)\left[1-\frac{p-b-2 A}{s}+\frac{1}{2}\left[\frac{p-b-2 A}{s}-\max \left(\frac{2 A-g+p}{s+\Delta}, 0\right)\right]\right]-k
$$

When $\Delta$ is positive, this expression is strictly increasing in $A$, and, thus, it is optimal to increase $A$ up to an opaque marketing strategy. Meanwhile, when $\Delta$ is negative, it is increasing for low values of $A$, but when $A \geq \frac{1}{2}(g-p)$, it decreases. Therefore, the optimal marketing choice in this case is $A^{*}=\frac{1}{2}(g-p)$-that is, when $T_{A N}=0$. Profits are simplified to $\Pi_{I n t}=(p-c)\left[1-\frac{2 p-g-b}{2 s}\right]-k$, which leads to an optimal price $p_{I n t}^{*}=\frac{2(s+c)+b+g}{4}$ and $\Pi_{I n t}^{*}=\frac{(b+b+2(s-c))^{2}}{16 s}-k$. Note that the feasibility of this solution requires $T_{B A}^{* I n t}=\frac{2(s+c)-b-g}{4 s}=\frac{1}{2}+\frac{2 c-b-g}{4 s} \in(0,1)$, which is satisfied if $s$ is sufficiently high. This discussion, together with Corollary 5 , can be summarized in the following result.

Proposition 6 Intermediate marketing is optimal in the linear-uniform case with observable investment only if low-type consumers are sufficiently sensitive to match quality $(s \gg 0)$ and more sensitive than high-type consumers $(\Delta<0)$.

This proposition states that a necessary condition for intermediate marketing to be optimal is that high-value customers are relatively insensitive to quality $(\Delta<0)$. Intuitively, when $\Delta<0$, the ex-post valuations induced by an intermediate strategy (that is the value of good matches for lower types, and average valuations for higher types) might all be fairly similar, so that a single price allows the monopolist to extract much of the surplus.

Note that assuming a higher or lower sensitivity to quality for high $\theta$ are both plausible alternatives, depending on the setting. For example, if consumers have similar preferences but vary in income, then wealthier (high-value) consumers are also more sensitive to quality. ${ }^{11}$ However, one could also imagine that aficionados/extremists are relatively insensitive; for example, a sciencefiction fanatic might both have a higher average valuation and be relatively insensitive to quality compared to an occasional viewer, who would gain only by watching a film that is a good match.

\footnotetext{
${ }^{11}$ This is the standard model of vertical differentiation, as articulated, for example, in Tirole (1988) p.96.
} 
Comparing alternative marketing strategies. Once one has characterized optimal profits and feasibility conditions for all the possible different regimes (intermediate above, and transparent and opaque in Appendix A), we can compare them and choose the highest feasible profit among them. Figure 2 illustrates this for a particular choice of parameters. It shows how the optimal marketing and investment strategies vary with $s$ and $c$, when $b=1, g=3, \Delta=-0.5$ and $k=0.2$.

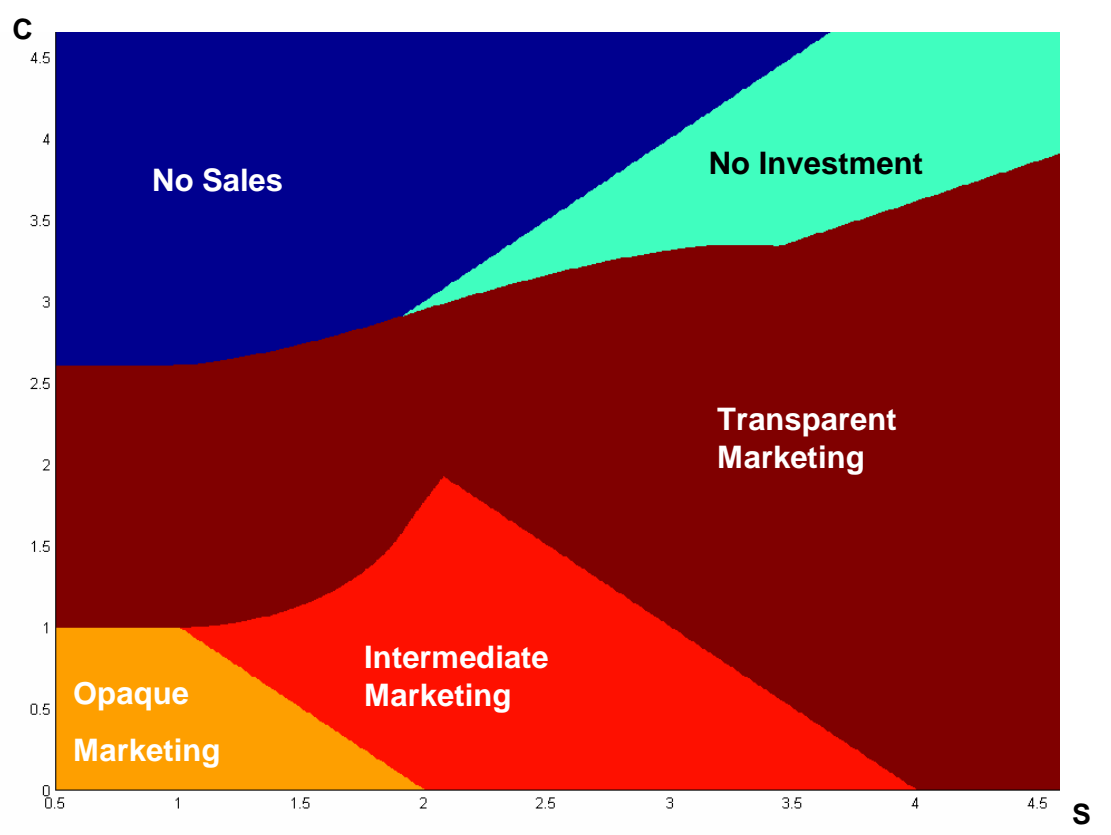

Figure 2: Marketing and Investment strategies with observable investment.

First, it is clear that when $c$ increases, the trade-off between higher margin and higher volume tilts in the direction of increasing margins. This implies that the firm should choose a more transparent marketing strategy. This can also be easily formalized by comparing the derivatives with respect to $c$ of the profit functions of each of the marketing strategies. For example, when $s=1.5$, then the marketing strategy changes from opaque, to intermediate, to transparent, and, finally, the firm would make no sales as $c$ increases (a shift up in the graph). Note that in regions where both $s$ and $c$ are relatively high, in equilibrium, the firm sells a relatively low quantity: since investment is a fixed cost, the firm prefers not to invest. In this case, since $s$ is high, it can still make sales to high $\theta$ consumers, but in this region, since consumers are certain of bad matches, the marketing strategy is irrelevant. 
Fixing $c$, increasing $s$ increases the dispersion in the valuations of different types of agents. As suggested by Corollary 5 and Proposition 6, intermediate marketing is optimal only when $s$ is sufficiently high, so that there is dispersion in valuations of different types of agent, who, therefore, choose different assessment strategies. Note that while increasing $s$ continues to increase such dispersion in valuations, for high enough values of $s$ (in particular for $s>2$ ), bad matches for the highest types are more valuable than good matches for lower types. When $s$ is high enough, therefore, the firm can discriminate between consumers and induce different behaviors with a transparent marketing strategy (with the highest type buying regardless of the realized match and lower types buying only after observing that the match is good); however, for lower $s$, differing assessment behavior is possible only with intermediate marketing. Moreover, assessment is a deadweight loss in this environment. As a result, for high enough values of $s$, transparent marketing is preferred to intermediate marketing.

Note that Corollary 5 implies that when consumers are homogeneous, the marketing strategy has to be extreme (transparent or opaque). If the firm could perfectly discriminate among heterogeneous consumers, it might choose the same extreme marketing strategy for all of them (albeit with different prices). Surprisingly, if the firm were then forced not to discriminate, intermediate marketing could be optimal. ${ }^{12}$ As a consequence, a firm that served two markets and employed the same marketing strategy in each, could choose a different marketing strategy if these two markets were integrated.

\section{Unobserved Investment}

So far, we have assumed that consumers directly observe the level of investment. However, sometimes it is not observable, and the firm may not be sufficiently established to commit to a given quality standard through reputation. In this case, consumers that assess and buy conditionally play an additional role: namely, that they act as quality monitors for those that buy unconditionally. Marketing can act as a form of indirect commitment: By inducing the right number of consumers to verify, the firm will invest in quality.

We now adapt the model and suppose that consumers do not observe the firm's investment level. Consumer behavior depends on the actual price and quantity, as above; however, it depends

\footnotetext{
${ }^{12}$ When the firm can discriminate, for each $\theta$, it can choose (i) either an opaque strategy with an optimal $p=$ $\frac{b+s \theta+g+(s+\Delta) \theta}{2}$ and earn $(p-c)$; or (ii) a transparent one at $p=g+(s+\Delta) \theta$ and earn $\frac{p-c}{2}$. Trivially, if $b>c$, the firm prefers an opaque strategy for all types. However, when discrimination is not possible, as can be seen in Figure 2, for example, at $c=0.5<1=b$, any marketing strategy (and, in particular, an intermediate one) can be optimal.
} 
on anticipated (rather than actual) investment. That is, $T_{B N}, T_{B A}$ and $T_{A N}$ will be functions of $\left(A, p, x^{e}\right)$ where $x^{e}$ represents the consumers' expectation of firm behavior. In equilibrium, consumers will accurately anticipate the firm's investment.

As in Section 3.2, the firm's problem is still to choose $A, p$ and $x$ in order to maximize profits, which are given by:

$$
\Pi=p S-c(S)-x,
$$

where the sales $S$ depend on $T_{B N}, T_{B A}$ and $T_{A N}$ and through them on $\left(A, p, x^{e}\right)$. As already mentioned, in equilibrium $x^{e}=x$. Thus, in equilibrium, it is as if there were an additional "incentivecompatibility" constraint: The firm must have no desire to choose a different investment level from the expected one. Note that the purchase behavior of consumers who buy without assessment (or regardless of the outcome) and of consumers who never buy are based on expected investment and are entirely unaffected by the firm's actual investment. The firm's actual investment affects only the purchase of those who assess and condition their purchase on the realization. Thus, to sustain an investment $x^{*}$, the firm must be optimizing with respect to those who are assessing:

$$
x^{*}=\arg \max _{x}(p-c)\left(\gamma(x) 1_{T_{B A}>T_{A N}} \int_{T_{A N}}^{T_{B A}} f(\theta) d \theta\right)-x
$$

There are a couple of consequences. First, note that Proposition 3 also applies when investment is unobservable, since the deviations suggested in its proof would not change the consumer behavior, and, so, would not change the level of investment in equilibrium. Second, and perhaps more directly, when investment is unobserved, if a firm chooses an opaque strategy, then sales do not depend on investment (the right-hand side of Equation (15) is 0). As a consequence, the firm would not invest and consumers would anticipate this, proving the following result.

Proposition 7 When investment is unobservable, opaque marketing $(A=\infty)$ is strictly optimal only if there is no investment $(x=0)$.

This proposition is central to understanding the second mechanism described in the introduction. It is at the heart of the idea that the marketing strategy is employed as a means of committing to investment. 
Next, we prove a couple of results. The first one compares different equilibria when investment is not observable. The second compares the case where investment is observed to where it is not.

When the firm's investment cannot be observed, in principle, there may be multiple equilibria. For example, suppose that $\gamma(x)=0$, and consider a set of parameters for which there exists an equilibrium with positive quality investment and some consumers assessing. For this same case, there also exists another equilibrium in which there is no investment: If consumers believe that the firm makes no investment, they will be certain of a bad match; therefore, they would have no reason to assess the good (even if it is costless to do so). Given this, the firm, indeed, has no reason to investment.

The following result shows that taking the observed choices as fixed, all consumers and the firm agree on the ranking among multiple equilibria. This leads to a natural equilibrium selection criterion: We assume that for a given price and marketing strategy, the equilibrium played is the Pareto dominant one. This criterion is later used for the characterization and comparative statics of Section 6.

Proposition 8 Given fixed values of $A$ and $p$, for any two equilibria with different investment levels, there is one that Pareto dominates the other. That is, the equilibrium with higher profits is also the one preferred by all consumers.

Proof. Suppose that there are two equilibria 1 and 2, and denote profits, quantity sold and investment by $\Pi_{i}, S_{i}$ and $x_{i}$ for $i=1,2$, respectively, with $x_{1}>x_{2}$.

First, note that in equilibrium 1 a consumer could behave as in equilibrium 2 , and achieve at least the same utility as in equilibrium 2. Thus, given that $x_{1}>x_{2}$, each consumer is at least as well off in equilibrium 1 as in equilibrium 2.

Second, note that $S_{1} \geq S_{2}$. The logic here is as follows: If a given type $\theta$ assesses in equilibrium 2 , then in equilibrium 1 she will either assess or buy without assessment; if she buys without assessment in 2 , then she would do the same in 1 . In both cases, since $x_{1}>x_{2}$ sales in 1 can be no lower than sales in 2 .

Finally, we show that $\Pi_{1} \geq \Pi_{2}$. Suppose, for contradiction, that $\Pi_{2}>\Pi_{1}$. Then, in equilibrium 1, given the assessment behavior of consumers described in the paragraph above, the firm would have a profitable deviation to invest $x_{2}$. Sales under this deviation, $S_{D}$, can be no lower than the 
sales in equilibrium 2: the investment is the same and consumers are more prone to assess and buy. Therefore, deviation profits $\Pi_{D}=p S_{D}-x_{2} \geq p S_{2}-x_{2}=\Pi_{2}>\Pi_{1}$, which provides the contradiction.

Our final result contrasts the case where investment is observed and where it is not.

Proposition 9 If transparent marketing $(A=0)$ is optimal for a firm when investment is observable, then it is also optimal when investment is not observable.

Proof. When $A=0$, consumer behavior is entirely determined by $b(\theta), g(\theta)$ and $p$. A consumer $\theta$ buys unconditionally if $p<b(\theta)$, buys conditionally if $b(\theta)<p<g(\theta)$, and never buys if $p>g(\theta)$. Thus, for a given $p$, when $A=0$, consumer behavior is independent of the investment $x$.

Take the optimal choice $\left(A^{*}=0, p^{*}, x^{*}\right)$ by the firm when investment is observable. $x^{*}$ is the solution to the maximization of $\left(p^{*}-c\right) S(x)-x$, where $S(x)$ is given by (7) evaluated at $A^{*}=0$ and $p^{*}$. Note that when $A^{*}=0$, given the above, $T_{B A}, T_{A N}$, and $T_{B N}$ do not depend on $x$. So, one can easily see that this program is equivalent to the one in (15). It follows, therefore, that $\left(A^{*}=0, p^{*}, x^{*}\right)$ is feasible when investment is unobservable, as well. Trivially, this is, then, the solution to the unobservable investment case.

The main message of this section is that when quality investment is unobservable, the only incentive of the firm to invest comes from the consumers that verify quality and buy conditionally. This is suggestive that, compared to the case where the firm can commit to quality, the inability to commit leads to higher transparency. Again, to fully characterize equilibrium and to run some comparative statics, we use linear utility functions and a uniform distribution of consumer types.

\section{The Linear-Uniform Case with Unobserved Investment}

We can follow the analysis in Section 4 and, now, consider the case where consumers do not observe investment. We use Proposition 8 to select the Pareto optimal equilibrium among the multiple ones that may arise for a given choice of $A$ and $p$ (which are observed by all consumers and chosen by the firm).

Recall that, for the linear-uniform case, we assume a simple investment function, whereby with no investment a bad match is realized with certainty, but if the firm invests at cost $k$, the probability

of a good match is $\frac{1}{2}$. The condition that determines the investment level, Equation (15), yields that there is investment if and only if 


$$
\frac{1}{2}(p-c)\left(T_{B A}-T_{A N}\right) 1_{T_{B A}>T_{A N}} \geq k
$$

That is, the firm invests only if the costs of doing so are smaller than the profits generated from those consumers buying conditionally.

As in Section 4, when the firm makes no investment, it earns $\Pi_{N I}^{*}=\max \left\{0, \frac{(b-c+s)^{2}}{4 s}, b-c\right\}$. Suppose that the firm invests in quality in equilibrium; following Proposition 7, it cannot be choosing an opaque strategy. Thus, if the firm does invest, it does so while choosing either an intermediate or a transparent marketing policy. As in Section 4, we can consider maximized profits under these marketing strategies, recognizing that (16) may bind. We conduct this analysis in Appendix B, and this allows us to compare these different strategies.

Comparing alternative marketing strategies. In parameter ranges in which the investment incentive constraint (16) does not bind, all results must be identical to those in Section 4. Further, the first part of Proposition 6 (that the optimality of intermediate marketing requires $s>>0$ ) applies for a firm with unobservable investment. This is easily verified, since if $s=0$, with intermediate marketing either $T_{B A}^{I n t}=\infty$ or $T_{B A}^{I n t}=T_{A N}^{I n t}$, both these outcomes suggest that intermediate marketing cannot be optimal.

Outside of these parameter ranges, however, the remaining results need not be true. In particular, when $\Delta>0$, for example, at $b=1, g=3, s=2, \Delta=1, k=0.2$ and $c=0.1$, it can be easily verified that intermediate marketing is preferred.

Figure 3 illustrates optimal marketing strategies at the same parameter values as Figure $2(b=1$, $g=3, \Delta=-0.5$ and $k=0.2)$. 


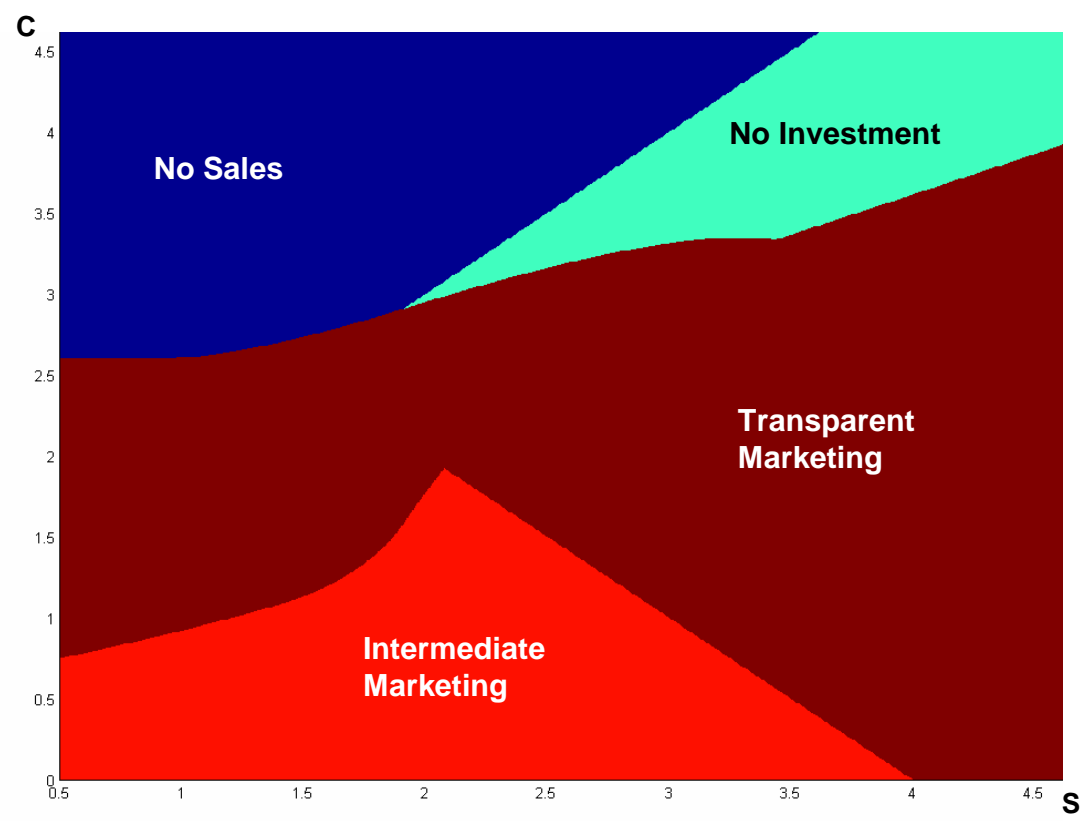

Figure 3: Optimal investment and marketing strategies with unobservable investment.

Comparing the optimal strategies in the two figures, when investment is not observable, opaque marketing is never optimal, as proven in Proposition 7. In the parameter region for which opaque marketing is optimal when investment is observable, then under non-observable investment, both transparent marketing and intermediate marketing can become optimal. In particular, for low values of $s$ and $c$ (where the profit per unit earned is relatively high, so the IC condition is easier to satisfy), intermediate marketing is preferred but for higher values of $c$, where the firm charges a higher price and sells fewer units, it is more difficult to satisfy (16) under intermediate marketing, and transparent marketing is preferred.

\section{Conclusions}

We have presented a simple framework in which marketing strategies interact with quality provision and pricing policies in an environment where agents need to exert effort to learn their valuation of a good and are heterogeneous in their tastes. Marketing strategies are modeled in a reduced form in which the firm can make it more or less difficult for consumers to learn their true valuation for the good. Quality provision is modeled as a productive effort that improves the probability of a good match between consumers and the good. 
While a first intuition might be that releasing more information should increase firm profits, a deeper reflection shows that this might not be the case. The main force at work is whether the firm prefers to provide little information and have a low markup with a high volume of sales or to provide more information and have a high markup with lower sales to a selected niche of consumers. If the firm provides little information - that is, if it is too costly for consumers to know their true valuation for the good - it may sell to all consumers at a low price. Meanwhile, if the firm provides cheap information, consumers learn whether they would enjoy the good, and the firm may charge a high price and sell only to those encountering a high valuation.

With heterogeneous consumers, the firm may decide on an intermediate marketing strategy to sort different types of consumers into different regimes. This may happen even when, in isolation, each consumer would face the same extreme marketing. Summarizing, we show that both informative advertising and obfuscation strategies can be the result of optimal behavior by firms and, further, that extreme marketing strategies may not always be optimal. The interior marketing strategy can be considered as a (non-price) means of discriminating between consumers, as suggested in Proposition 3.

In addition to this trade-off of quantity vs. markup, if the firm cannot publicly commit to providing high quality, a further effect is at work, as highlighted in Proposition 7. Here, a way to indirectly commit to quality is to choose a sufficiently transparent policy that induces consumer assessment and disciplines the firm. In particular, there are cases with intermediate marketing in which some consumers verify the quality of the good and buy conditionally, while others buy unconditionally. In this case, there is an externality at work: The consumers that verify the quality of the good force the firm to exert effort in quality provision that also benefits consumers who buy unconditionally.

The paper has considered a monopoly provider. In a competitive market, information provision plays an additional role - it softens price competition by creating some product differentiation, as in Meuer and Stahl (1994) and Hotz and Xiao (2007). Therefore, this differentiation motive pushes towards more transparent marketing policies. However, and particularly if firms offer exante differentiated products, the effects highlighted in this paper should still play a role. A full analysis of these issues lies outside the scope of this paper. 


\section{References}

[1] Anand, Bharat and Ron Shachar (2005) "Advertising the Matchmaker", working paper

[2] Anderson, Simon P. and Regis Renault (2006): "Advertising Content," American Economic Review, 96(1), pp. 93-113.

[3] Bagwell, Kyle (2007): "The Economic Analysis of Advertising," Chapter 28 in Handbook of Industrial Organization Vol. 3 eds Armstrong, M. and R. H. Porter, North Holland, pp. 17011844.

[4] Bar-Isaac, Heski, Guillermo Caruana and Vicente Cuñat (2008): "Information gathering externalities in product markets" working paper.

[5] Bergemann, Dirk and Juuso Välimäki (2002), "Information Acquisition and Efficient Mechanism Design," Econometrica, 70, pp. 1007-1034.

[6] Creane, Anthony (2008), "A note on welfare-improving ignorance about quality," Economic Theory, 34, pp. 585-590.

[7] Cremer, Jacques and Fahad Khalil (1992), "Gathering Information before Signing a Contract," American Economic Review, 82, pp. 566-578.

[8] Cremer, Jacques, Fahad Khalil and Jean-Charles Rochet (1998a): "Contracts and Productive Information Gathering," Games and Economic Behavior, 25, pp. 174-193.

[9] Cremer, Jacques, Fahad Khalil, and Jean-Charles Rochet (1998b), "Strategic Information Gathering before a Contract is Offered," Journal of Economic Theory, 81,pp. 163-200.

[10] Ganuza, Juan-José and José S. Penalva (2006): "On Information and Competition in Private Value Auctions," UPF Working Paper \#937.

[11] Hotz, V. J. and M. Xiao (2007): "Strategic Information Disclosure: The Case of MultiAttribute Products with Heterogeneous Consumers,"mimeo, UCLA.

[12] Johnson, Justin P. and David P. Myatt (2006): "On the Simple Economics of Advertising, Marketing, and Product Design," American Economic Review, 96(3).

[13] Lewis, Tracy R. and David E. M. Sappington (1994): "Supplying Information to Facilitate Price Discrimination," International Economic Review, 95(2), pp. 309-327.

[14] Lewis, Tracy R. and David E. M. Sappington (1997), "Information Management in Incentive Problems," Journal of Political Economy, 105, pp. 796-821.

[15] Matthews, Steven A. and Nicola Persico (2005): "Information Acquisition and the Excess 
Refund Puzzle," Penn Institute for Economic Research, working paper \#05-015.

[16] Meuer, Michael and Dale O. Stahl II (1994): "Informative Advertising and Product Match," International Journal of Industrial Organization, 12(1), pp. 1-19.

[17] Saak, Alexander E. (2006): "The optimal private information in single unit monopoly," Economics Letters, 91, 267-272

[18] Sun, Monic Jiayin (2007): "Disclosing Multiple Product Attributes" working paper, Boston University

[19] Tirole, Jean (1988): The Theory of Industrial Organization, MIT Press.

[20] Zettelmeyer, Florian (2000): "The Strategic Use of Consumer Search Cost," Haas, Berkeley, working paper.

\section{A Opacity and Transparency in the Linear-Uniform Case with Observ- able Investment}

Here, we characterize the optimal pricing strategies and profits under the assumption that the firm invests, first in the case that the firm chooses opaque marketing, and, next, transparent marketing.

\section{A.1 Opaque marketing}

Under opaque marketing $(A=\infty)$, we have that $T_{A N}=1$ and $T_{B A}=0$. Thus, the firm's profits from Equation (9) can be rewritten as

$$
\Pi_{O p}=(p-c)\left(1-T_{B N}\right)-k .
$$

where $T_{B N}=\max \left(\min \left(\frac{2 p-g-b}{2 s+\Delta}, 1\right), 0\right)$. Maximizing this expression with respect to $p$, leads to either a $T_{B N}$-interior optimal price of $p_{O p}^{*}=\frac{b+g+\Delta+2(c+s)}{4}$ (and profits of $\Pi_{O p}^{*}=\frac{(b+g+2(s-c)+\Delta)^{2}}{2(2 s+\Delta)}-k$ ), or a corner $T_{B N}^{*, O p}=0$ solution with $p_{O p}^{*}=\frac{g+b}{2}$ (and profits of $\left.\Pi_{O p}^{*}=\frac{g+b}{2}-c-k\right)$. Note that a corner $T_{B N}=1$ solution is always suboptimal, as no sales are realized, but a $k$ investment cost is incurred.

\section{A.2 Transparent marketing}

Under transparent marketing $(A=0), T_{B A} \geq T_{B N} \geq T_{A N}$, which simplifies the firm's profits from Equation (9) to:

$$
\Pi_{T r}=(p-c)\left[\left(1-T_{B A}\right)+\frac{1}{2}\left(T_{B A}-T_{A N}\right)\right]-k
$$

where $T_{A N}=\max \left(\min \left(\frac{p-g}{s+\Delta}, 1\right), 0\right)$ and $T_{B A}=\max \left(\min \left(\frac{p-b}{s}, 1\right), 0\right)$. As one can see, sales are a piecewise linear function of $p$. Thus, the optimal price expression differs depending on which part of the piecewise function is the relevant one.

First, we consider the cases in which the price is such that no consumer is excluded $\left(T_{A N}=0\right)$. Choosing $p \leq b$ and selling regardless of the realization cannot be optimal, as the firm would prefer then not to invest in quality at all. Next, if the optimal price is such that $T_{B A} \in(0,1)$, then profits can be written as $(p-c)\left[1-\frac{p-b}{2 s}\right]-k$, the maximization problem yields $\Pi_{T r}^{*}=\frac{(b-c+2 s)^{2}}{8 s}-k$, an this happens as long as $b<p_{T r}^{*}=\frac{b+c+2 s}{2}<\min \{g, b+s\}$. Finally, the firm can choose $p=g$, selling only in the case of a good match-realization, and earning $\Pi_{T r}=\frac{g-c}{2}-k$.

Alternatively, the firm can choose a price that excludes some consumers $\left(T_{A N}>0\right)$. In the region where $T_{B A}<1$, profits are given by $(p-c)\left[1-\frac{1}{2} \frac{p-b}{s}-\frac{1}{2} \frac{p-g}{s+\Delta}\right]$. Maximizing with respect to $p$ yields 
$p_{T r}^{*}=\frac{1}{2} \frac{\Delta(b+c+2 s)+s(b+g+2 c+2 s)}{2 s+\Delta}$ and profits of $\Pi_{T r}^{*}=\frac{(\Delta(b-c+2 s)+s(b+g-2 c+2 s))^{2}}{8 s(s+\Delta)(2 s+\Delta)}-k$. This case requires $p_{T r}^{*} \in(g, \min \{b+s, g+s+\Delta\})$. Finally, we consider the case with $T_{B A}=1$. Profits are $(p-c) \frac{1}{2}\left(1-\frac{p-g}{s+\Delta}\right)-k$, which are maximized at $p_{T r}^{*}=\frac{c+s+g+\Delta}{2}$ the maximization in this case yields $\Pi_{T r}^{*}=\frac{(g-c+s+\Delta)^{2}}{8(s+\Delta)}-k$ so long as $\max \{g, b+s\}<p_{T r}^{*}=\frac{c+s+g+\Delta}{2}<g+s$.

\section{B Intermediate and Transparent Marketing in the Linear-Uniform Case with Unobservable Investment}

\section{B.1 Intermediate marketing}

Following Proposition 3, an optimal intermediate marketing strategy requires $1>T_{B A}>T_{A N} \geq 0$. As in Section 4, we can use Equation (13) to express profits:

$$
\Pi_{\text {Int }}=(p-c)\left[1-\frac{p-b-2 A}{s}+\frac{1}{2}\left[\frac{p-b-2 A}{s}-\max \left(\frac{2 A-g+p}{s+\Delta}, 0\right)\right]\right]-k .
$$

However, here the firm faces the additional constraint stated in (16):

$$
(p-c)\left[\frac{p-b-2 A}{s}-\max \left(\frac{2 A-g+p}{s+\Delta}, 0\right)\right]>2 k .
$$

Note that the left-hand side of this constraint is linear and decreasing in $A$. There are a number of possibilities to be considered.

First, Equation (20) might not be binding; then, the analysis of Section 4 applies and so the firm would choose $A^{*}=\frac{g-p}{2}, p_{I n t}^{*}=\frac{2(s+c)+b+g}{4}$ and maximized profits would be $\Pi_{I n t}^{*}=\frac{(b+g+2(s-c))^{2}}{16 s}$. Note that the feasibility of this solution requires $1>T_{B A}^{*, I n t}=\frac{2(s+c)-b-g}{4 s}>0$ and the new constraint that $\frac{4 s^{2}-(b+g-2 c)^{2}}{16 s}>k$.

Alternatively, Equation (20) might bind. Here, there are two cases depending, on whether $\frac{g-p}{2} \gtrless A$. In the case that $\frac{g-p}{2}<A$, solving for $A$ as a function of $p$ when (20) binds (and no other condition binds) substituting into the profit function and simplifying, we can obtain $p_{I n t}^{*}=\frac{2(c+s)+\Delta+B+G}{4}, \Pi_{I n t}^{*}=$ $\frac{1}{8} \frac{(b+g-2 c+2 s+\Delta)^{2}}{2 s+\Delta}-\frac{\Delta}{2 s+\Delta} k$ and $A^{*}=\frac{1}{2} \frac{(p-b) \Delta+(g-b) s}{2 s+\Delta}-\frac{s(s+\Delta)}{(p-c)(2 s+\Delta)} k$. Note that we also require that $1>$ $T_{B A}>T_{A N} \geq 0$, which can be written as:

$1>T_{B A}=\frac{1}{2} \frac{2 c+2 s+\Delta-b-g}{2 s+\Delta}+\frac{8(s+\Delta)}{(2 s+\Delta)(b+g-2 c+2 s+\Delta)} k>\frac{1}{2} \frac{2 c+2 s+\Delta-b-g}{2 s+\Delta}-\frac{8 s}{(2 s+\Delta)(b+g-2 c+2 s+\Delta)} k \geq 0$.

A final case is that $(20)$ binds and that $T_{A N}=0$, which requires that $\frac{g-p}{2}>A$. In this case, when (20) binds, $\frac{p-c}{2} T_{B A}=k$, and so $T_{B A}=\frac{2 k}{p-c}$ and (substituting in for $T_{B A}$ ) $A=\frac{p-b}{2}-\frac{s k}{p-c}$. Substituting into the profit function, we obtain $(p-c)\left(1-\frac{2 k}{p-c}+\frac{k}{p-c}\right)$, and so the firm sets the price as high as possible, subject to constraints. Thus, the "new constraint" $T_{A N} \geq 0$ binds and we require $\frac{g-p}{2}=A$, which yields the two solutions $p=\frac{1}{4}\left(b+g+c \pm \sqrt{b^{2}-4 b c+2 b g+4 c^{2}-4 c g+g^{2}+16 k s}\right)$. One can substitute back to obtain maximized profits in this case.

\section{B.2 Transparent marketing}

The solutions computed in Appendix A.2 are also the solutions here, because, as the proof of Proposition 9 shows, they also satisfy the incentive-compatibility constraint. 